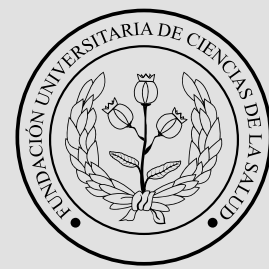

FUCS
Re

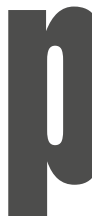

\title{
Impacto del COVID-19 en la salud mental: revisión de la literatura
}

Carlos A. Alvarez Jorge I. Toro ${ }^{b}$

\section{Impact of COVID-19 on mental health: a literature review}

\begin{abstract}
${ }^{a}$ Docencia Universitaria. Psicólogo. Coordinador de investigaciones Docente del programa de psicología, Grupo de Investigación en Psicología, Salud, Administración y Docencia - GIPSAD. Fundación Universitaria de Ciencias de la Salud. Bogotá DC, Colombia.

${ }^{b}$ Psicología Clínica, Grupo de Investigación en Psicología, Salud, Administración y Docencia-GIPSAD, Fundación Universitaria De Ciencias de la Salud. Bogotá DC, Colombia.
\end{abstract}

\section{R E S U M EN}

Introducción: el 12 de diciembre de 2019 en Wuhan, China, se reportó el primer caso de COVID-19, que después se extendió y alcanzó la categoría de pandemia mundial, obligando a los gobiernos a tomar medidas de salud pública urgentes; lo que ha implicado la aparición o complicación de los problemas de salud mental en la población. Objetivo: describir el impacto del COVID-19 en la salud mental a partir de la revisión de la literatura científica. Materiales y métodos: se revisó la literatura consultando 9 bases de datos con los términos de búsqueda "COVID-19" y "mental" y el operador booleano "AND". Los artículos fueron revisados y sintetizados con la ayuda de una matriz de 5 pasos y evaluados con las pruebas STROBE y CASPe. Un total de 43 artículos fueron seleccionados. Resultados: se encontró que China con 21 estudios (46,7\%) presentó la mayor producción, utilizando diversos tipos de instrumentos aplicados de modo remoto. Las muestras fueron tomadas en momentos diferentes del brote (fase inicial, pico de brote y después del pico), desde diciembre 2019 hasta abril de 2020, en especial con diseños transversales $(90.7 \%)$. El impacto psicológico se agrupó en las categorías de afectos negativos, afectos positivos, pensamientos negativos, ansiedad, depresión, estrés, alteraciones del comportamiento, consumo de sustancias psicoactivas, síntomas fisiológicos, alteraciones del sueño, impacto psicológico y cambios positivos en el bienestar. Conclusiones: se evidenciaron impactos del brote del COVID-19 en la salud mental. No se hallaron estudios colombianos.

Palabras clave: COVID-19, infecciones por coronavirus, salud mental, pandemias.

(C) 2021 Fundación Universitaria de Ciencias de la Salud - FUCS. Este es un artículo Open Access bajo la licencia CC BY-NC-ND (http://creativecommons.org/licenses/by-nc-nd/4.0/).

INFORMACIÓN DEL ARTÍCULO

Historia del artículo:

Fecha recibido: abril 13 de 2021

Fecha aceptado: mayo 13 de 2021
Autor para correspondencia:

Sr. Carlos A. Alvarez

caalvarez@fucsalud.edu.cl
DOI

10.31260/RepertMedCir.01217372.1180 
Introduction: the first case of COVID-19 was reported in Wuhan, China on December 12 2019, the disease spread rapidly becoming a global pandemic; governments were forced to take urgent public health measures; causing the development or complication of psychological issues in the general population. Objective: to describe the impact of COVID-19 on mental health based on a review of the scientific literature. Materials and Methods: the literature review consisted of a search in nine databases using the terms "COVID-19" and "mental" and Boolean operator "AND". Articles were reviewed and synthetized based on a 5-step matrix and evaluated using the STROBE and CASPe scales. A total of 43 articles were selected. Results: the highest number of studies (21- 46.7\%) was found in China, using diverse types of remotely applied instruments. Samples were taken at different times of the outbreak (initial stage, outbreak peak and post-peak), from December 2019 to April 2020 , relying mostly on cross-sectional studies $(90.7 \%)$. The psychological impact was grouped in the following categories: negative affect, positive affect, negative thoughts, anxiety, depression, stress, behavioral alterations, use of psychoactive substances, physiologic symptoms, sleep disturbances, psychological impact and positive changes in wellbeing. Conclusions: various impacts of the COVID-19 outbreak on mental health were evidenced. No studies on this matter were found in Colombia.

Key words: COVID-19, coronavirus infections, mental health, pandemics

(C) 2021 Fundación Universitaria de Ciencias de la Salud - FUCS. This is an open access article under the CC BY-NC-ND license (http://creativecommons.org/licenses/by-nc-nd/4.0/).

\section{INTRODUCCIÓN}

El 12 de diciembre de 2019 se reportó el primer caso de la nueva neumonía por coronavirus en Wuhan, China. El 12 de enero de 2020 la Organización Mundial de la Salud (OMS) la denominó "Coronavirus Disease 2019", COVID-19. ${ }^{1}$ La nueva enfermedad COVID-19 se extendió por el orbe hasta generar una pandemia global ${ }^{2}$ y se declaró emergencia internacional de salud pública por la OMS. ${ }^{3}$

El COVID-19 tiene un periodo de incubación largo, es de fácil transmisión, tiene una tasa de mortalidad importante4 y hasta el momento se mantiene sin tratamiento específico ${ }^{1}$, razones para crear gran pánico y ansiedad (Elbay, Kurtulmuş, Arpacıoğlu \& Karadere, 2020). Estos riesgos han llevado a los gobiernos a implementar diferentes tipos de medidas urgentes de salud pública, entre ellas las de distanciamiento social y confinamiento. La pandemia ha implicado la aparición o complicación de los problemas de salud mental en la población, con especial énfasis en ciertos subgrupos, temática que empieza a ser objeto de investigación en diversos países. Por ejemplo, algunos estudios indican que el personal sanitario, en especial de primera línea, experimentó una alta presión psicológica ${ }^{1,5}$ y que la población en confinamiento que se restringió del contacto social experimentó emociones negativas y preocupaciones4 que disminuyeron su bienestar en momentos diferentes del brote.

Las medidas en salud pública se tomaron con base en la evidencia científica y más aún en tiempo de una nueva pandemia. Con este contexto se propone describir el impacto en la salud mental a causa del brote mundial del COVID-19, a través de la revisión de la literatura científica.

\section{MATERIALES Y MÉTODOS}

El estudio se enmarca en una revisión de literatura científica con el propósito de informar los resultados de investigaciones publicadas a raíz del brote del COVID-19. La revisión es parte integral de toda investigación, entre otras, por la utilidad para informar y desarrollar la práctica del conocimiento e invitar a la discusión en el trabajo académico. ${ }^{6}$

Se realizaron búsquedas electrónicas en nueve bases de datos (Pubmed-Medline, Lilacs, EBSCO Discovery Service, Scielo, ProQuest, J-STOR, Biblioteca Virtual en Salud, Science Direct y PsyInfo), siete de ellas consultadas desde el servidor de la biblioteca de la Fundación Universitaria de Ciencias de la Salud de Bogotá y 2 en la web abierta, realizadas a mediados de junio 2020. Los criterios de selección fueron artículos en inglés o español, investigación original en seres humanos, estudios con metodologías cuantitativas, cualitativas o mixtas, publicados entre 2019 y 2020. Se utilizaron los términos de búsqueda "COVID-19", Mental y el operador booleano "AND". Para la valoración de la calidad científica de los documentos se recurrió a las herramientas CASPe y STROBE. ${ }^{7}$

Se construyó una matriz de selección y evaluación de la información de 5 pasos para registrar, filtrar, organizar y analizar los datos extraídos de los documentos, lo cual exigió establecer los objetivos que perseguían los autores, el enfoque del estudio y las contribuciones de cada artículo para su análisis. ${ }^{8}$ Los filtros permitieron seleccionar un total de 43 documentos (tabla 1).

Se presenta el esquema que orienta el diseño metodológico y los filtros asumidos para la selección final de los estudios (figura 1). 
Tabla 1. . Artículos seleccionados en la revisión

1 Xing J, Sun N, Xu J, Geng S, Li Y.

2 Tull MT, Edmonds KA, Scamaldo KM, Richmond JR, Rose JP, Gratz KL.

3 Elbay RY, Kurtulmuş A, Arpacıoğlu S, Karadere E

4 Ahmed MZ, Ahmed O, Aibao Z, Hanbin S, Siyu L, Ahmad A.

5 Amerio A, Bianchi D, Santi F, Costantini L, Odone A, Signorelli C, Costanza A, Serafini G, Amore M, Aguglia A.

6 Bitan, D. T., Giron, A., Bloch, Y., Mayer, Y., shiffman, N., Y Mendlovic, Shlomo.

Chew NWS, Lee GKH, Tan BYQ, Jing M, Goh Y, Ngiam NJH, Yeo LLL, Ahmad A, Ahmed Khan F, Napolean

7 Shanmugam G, Sharma AK, Komalkumar RN, Meenakshi PV, Shah K, Patel B, Chan BPL, Sunny S, Chandra B, Ong JJY, Paliwal PR, Wong LYH, Sagayanathan R, Chen JT, Ying $\mathrm{Ng}$ AY, Teoh HL, Tsivgoulis G, Ho CS, Ho RC, Sharma VK

8 Choi, E.P.H.; Hui, B.P.H.; Wan, E.Y.F.

9 Costantini A, Mazzotti E

10 Gao J, Zheng P, Jia Y, Chen H, Mao Y, Chen S, Wang Y, Fu H, Dai J.

11 Germani A, Buratta L, Delvecchio E, Mazzeschi C.

12 Gómez-Salgado, J.; Andrés-Villas, M.; Domínguez-Salas, S.; Díaz-Milanés, D.; Ruiz-Frutos, C

13 Goodman-Casanova JM, Dura-Perez E, Guzman-Parra J, Cuesta-Vargas A, Mayoral-Cleries F.

14 Guo J, Feng XL, Wang XH, van IJzendoorn MH. Guo Q, Zheng Y, Shi J, Wang J, Li G, Li C, Fromson JA,

15 Xu Y, Liu X, Xu H, Zhang T, Lu Y, Chen X, Hu H, Tang Y, Yang S, Zhou H, Wang X, Chen H, Wang Z, Yang Z

16 Hong,Yan., Cao, H.,Leung, D., y Mak, Y

17 Huang $Y$, Zhao N.

Jin YH, Huang Q, Wang YY, Zeng XT, Luo LS, Pan ZY,

18 Yuan YF, Chen ZM, Cheng ZS, Huang $X$, Wang N, Li BH, Zi H, Zhao MJ, Ma LL, Deng T, Wang Y, Wang XH.

19 Johnson María Cecilia, Saletti-Cuesta Lorena, Tumas Natalia. Lai J, Ma S, Wang Y, Cai Z, Hu J, Wei N, Wu J, Du H, Chen

20 T, Li R, Tan H, Kang L, Yao L, Huang M, Wang H, Wang G, Liu Z, Hu S.

21 Lei L, Huang X, Zhang S, Yang J, Yang L, Xu M.

22 Liu CH, Zhang E, Wong GTF, Hyun S, Hahm HC.

23 Liu CY, Yang YZ, Zhang XM, Xu X, Dou QL, Zhang WW, Cheng ASK.

24 Liu S, Liu Y, Liu Y.

25 Liu X, Luo WT, Li Y, Li CN, Hong ZS, Chen HL, Xiao F, Xia JY.

26 Madani A, Boutebal SE, Bryant CR.

27 Odriozola-González P, Planchuelo-Gómez Á, Irurtia MJ, de Luis-García $R$.

28 Olufadewa, I., Adesina, M., Oladokun, B., Baru, A., Oladele, R., lyanda, T., Ajibade, O., Abudu, F.

29 Ozamiz-Etxebarria, Naiara et al.

30 Rossi R, Socci V, Pacitti F, Di Lorenzo G, Di Marco A, Siracusano A, Rossi A.

31 Smith L, Jacob L, Yakkundi A, McDermott D, Armstrong NC, Barnett Y, López-Sánchez GF, Martin S, Butler L, Tully MA.
Study of the mental health status of medical personnel dealing with new coronavirus pneumonia. Psychological Outcomes Associated with Stay-at-Home Orders and thePerceived Impact of COVID-19 on Daily Life.

Depression, anxiety, stress levels of physicians and associated factors inCovid-19 pandemics.

Epidemic of COVID-19 in China and associated Psychological Problems.

Covid-19 pandemic impact on mental health: a web-based cross-sectional survey on a sample of Italian general practitioners.

Fear of COVID-19 scale: Psychometric characteristics, reliability and validityin the Israeli population.

A multinational, multicentre study on the psychological outcomes andassociated physical symptoms amongst healthcare workers during COVID-19 outbreak.

Depression and Anxiety in Hong Kong during COVID-19.

Italian validation of CoViD-19 Peritraumatic Distress Index and preliminary data in a sample of general population.

Mental health problems and social media exposure during COVID-19 outbreak.

Emerging Adults and COVID-19: The Role of Individualism-Collectivism on Perceived Risks and Psychological Maladjustment.

Related Health Factors of Psychological Distress During the COVID-19 Pandemic in Spain.

Telehealth Home Support During COVID-19 Confinement for Community-Dwelling Older Adults With Mild Cognitive Impairment or Mild Dementia: Survey Study.

Coping with COVID-19: Exposure to COVID-19 and Negative Impact on Livelihood Predict Elevated Mental Health Problems in Chinese Adults.

Immediate psychological distress in quarantined patients with COVID-19and its association with peripheral inflammation: A mixed-method study.

The Psychological Impacts of a COVID-19 Outbreak on College Students in China: A Longitudinal Study.

Generalized anxiety disorder, depressive symptoms and sleep quality during COVID-19 outbreak in China: a web-based cross-sectional survey.

Perceived infection transmission routes, infection control practices, psychosocial changes, and management of COVID-19 infected healthcare workers in a tertiary acute care hospital in Wuhan: a cross-sectional survey.

Emociones, preocupaciones y reflexiones frente a la pandemia del COVID-19 en Argentina.

Factors Associated With Mental Health Outcomes Among Health Care Workers Exposed to

Coronavirus Disease 2019.

Comparison of Prevalence and Associated Factors of Anxiety and Depression Among People Affected by Versus People Unaffected by Quarantine During the COVID-19 Epidemic in Southwestern China.

Factors associated with depression, anxiety, and PTSD symptomatologyduring the COVID-19 pandemic: Clinical implications for U.S. young adultmental healt.

The Prevalence and Influencing Factors in Anxiety in Medical Workers Fighting COVID-19 in China: A Cross-Sectional Survey.

Somatic symptoms and concern regarding COVID-19 among Chinese collegeand primary school students: A cross-sectional survey.

Psychological status and behavior changes of the public during the COVID-19 epidemic in China. The Psychological Impact of Confinement Linked to the Coronavirus Epidemic COVID-19 in Algeria.

Psychological effects of the COVID-19 outbreak and lockdown amongstudents and workers of a

Spanish university.

"I Was Scared I Might Die Alone": A Qualitative Study on the Physiological and Psychological Experience of COVID-19 Survivors and the Quality of Care Received at Health Facilities.

Niveles de estrés, ansiedad y depresión en la primera fase del brote del COVID-19 en una muestra recogida en el norte de España.

Mental Health Outcomes Among Frontline and Second-Line Health Care Workers During the

Coronavirus Disease 2019 (COVID-19) Pandemic in Italy.

Correlates of symptoms of anxiety and depression and mentalwellbeing associated with COVID-19: a cross-sectional study ofUK-based respondents. 
32 Song, X., Fu, W., Liu, X., Luo, Z., Wang, R., Zhou, N., Yan, S., \& Lv, C.

33 Stanton R, To QG, Khalesi S, et al.

34 Steven Taylor, Caeleigh A. Landry, Michelle M. Paluszek, Thomas A. Fergus, Dean McKay, Gordon J.G. Asmundson.

35 Sun D, Yang D, Li Y, Zhou J, Wang W, Wang Q, Lin N, Cao A, Wang $\mathrm{H}$, Zhang $\mathrm{Q}$.

36 Sun N, Wei L, Shi S, Jiao D, Song R, Ma L, Wang H, Wang C, Wang Z, You Y, Liu S, Wang H.

37 Varshney M, Parel JT, Raizada N, Sarin SK.

38 Wang $\mathrm{C}$, Pan R, Wan X, Tan Y, Xu L, McIntyre RS, Choo FN, Tran B, Ho R, Sharma VK, Ho C.

39 Wang $\mathrm{H}$, Xia Q, Xiong Z, Li Z, Xiang W, Yuan Y, Liu Y, Li Z.

Wanqiu, T., Fengyi, H., Roger S. M., Li, J., Xiaojiang, J.,

40 Ling Zhang, Xinling, Z., Yiran, Z., Yirong, H., Xi, L., Zhisong, Z., Andre, L., Roger,H., Bach, T., Cyrus, H., y Wilson, T.

41 Wu K, Wei X.

42 Yang S, Kwak SG, Ko EJ, Chang MC

43 Yingfei Zhang, and Zheng Feei Ma.
Mental health status of medical staffin emergency departments during theCoronavirus disease 2019 epidemic in China.

Depression, Anxiety and Stress during COVID-19: Associations with Changes in Physical Activity, Sleep, Tobacco and Alcohol Use in Australian Adults.

Development and initial validation of the COVID Stress Scales.

Psychological impact of 2019 novel coronavirus (2019-nCoV) outbreak in health workers in China.

A Qualitative Study on the Psychological Experience of Caregivers of COVID-19 Patients.

Initial psychological impact of COVID-19 and its correlates in Indian Community: An online (FEEL-COVID) survey.

A longitudinal study on the mental health of general population during theCOVID-19 epidemic in China.

The psychological distress and coping styles in the early stages of the 2019 coronavirus disease (COVID-19) epidemic in the general mainland Chinese population: A web-based survey.

Is returning to work during the COVID-19 pandemic stressful? A study onimmediate mental health status and psychoneuroimmunity preventionmeasures of Chinese workforce.

Analysis of Psychological and Sleep Status and Exercise Rehabilitation of Front-Line Clinical Staff in the Fight Against COVID-19 in China.

The Mental Health Burden of the COVID-19 Pandemic on Physical Therapists.

Impact of the COVID-19 Pandemic on Mental Health and Quality of Life among Local Residents in Liaoning Province, China: A Cross-Sectional Study.

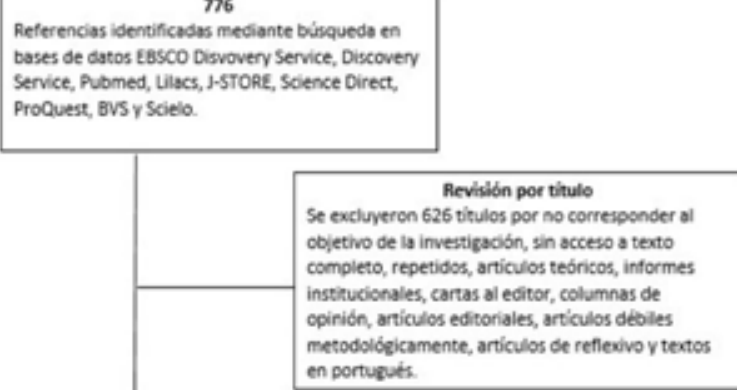

150 documentos.

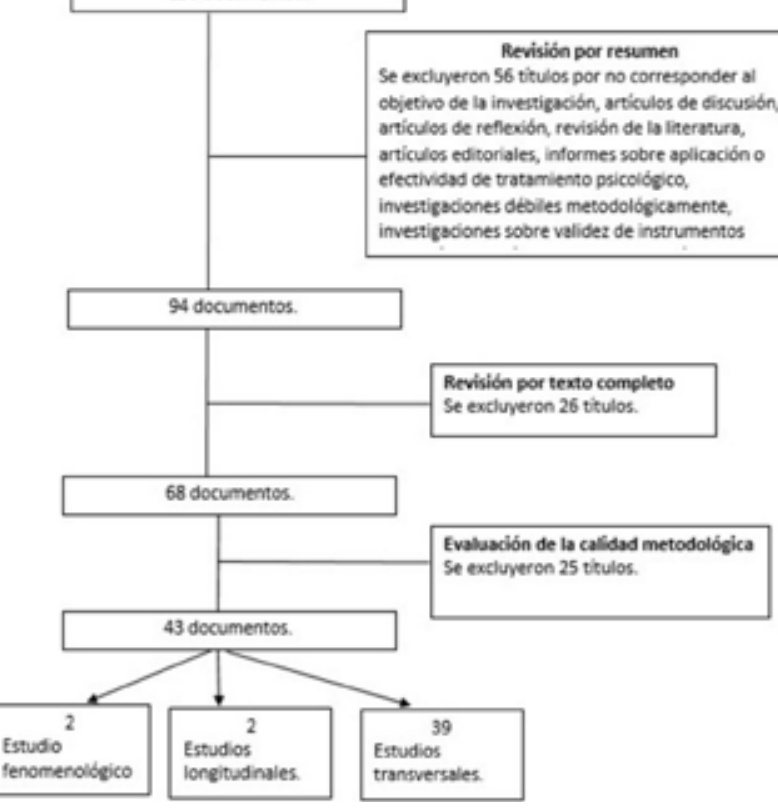

Figura 1. Proceso de selección y evaluación de estudios. Fuente: los autores.

\section{RESULTADOS}

El total de estudios seleccionados fueron 43, distribuidos por países encabezando la lista China con $21(46,7 \%)$, Italia $4(8,9 \%)$, España $4(8,9 \%)$, Estados Unidos $3(6,7 \%)$, seguidos por India $2(4,4 \%)$, Singapur (4,4\%) y Argelia, Argentina, Australia, Canadá, Corea del Sur, Turquía, Israel y Reino Unido cada uno con 1 (2,2\% cada uno), además de 1 estudio global $(2,2 \%)$.

Se usaron como instrumentos de investigación cuestionarios, inventarios, escalas, entrevistas, encuestas, listas de verificación y pruebas aplicados en modalidad remota principalmente (telefónica o en línea), realizados entre diciembre 2019 y abril 2020. Las muestras fueron tomadas en momentos diferentes del brote: fase inicial, pico de brote y después del pico. Se encontraron 3 estudios con enfoque cualitativo, 39 de enfoque cuantitativo y 1 de enfoque mixto. En cuanto a diseños de investigación 39 fueron transversales $(90,7 \%), 2$ longitudinales $(4,7 \%)$ y 2 fenomenológicos $(4,7 \%)$. Con las estrategias de búsqueda asumidas se pudo determinar que los hallazgos referentes a los impactos psicológicos del COVID-19 en la salud mental a nivel afectivo, cognitivo, comportamental y fisiológico, aparecen agrupados en las siguientes categorías: Afectos negativos que incluyen angustia ${ }^{9-12}$, conmoción $^{1}$, hostilidad ${ }^{13}$, incertidumbre ${ }^{12}$, miedo $^{1,12,13,14-16}$, sensación de indefensión ${ }^{17}$, sensibilidad interpersonal ${ }^{13}$, sentimientos de horror y aprehensividad ${ }^{17}$, sentimientos de impotencia ${ }^{18}$, sentirse abrumado y tenso ${ }^{10}$, sentirse de mal humor ${ }^{19}$, soledad vinculada al encierro ${ }^{12}$, terror $^{13}$, entre otros. ${ }^{14,20}$ Afectos positivos que incluyen bienestar ${ }^{21}$, calma $^{15}$ y tolerancia al estrés. ${ }^{22}$ 
Pensamientos negativos que incluyen rechazo inicial de la situación ${ }^{1}$, miedo a infectarse ${ }^{14}$, miedo al COVID-19 $9^{23}$, no dejar de pensar en la pandemia y de cómo protegerse a sí mismo ${ }^{19}$, preocupaciones financieras ${ }^{4}$, por familiares ${ }^{16}$, por morir en caso de contagio por COVID-19 ${ }^{9}$, acerca de la salud de la familia ${ }^{24}$, respecto al COVID-19 relacionadas con uno mismo, preocupaciones sobre COVID-19 para con familiares ${ }^{25}$, percepción de deterioro de la salud mental desde el inicio de la pandemia ${ }^{26}$ y de riesgo de infección. ${ }^{25}$

Ansiedad que incluyen estar nervioso ${ }^{24}$, propensión a la ansiedad $1,{ }^{26}$, experimentar con mayor intensidad por la salud4, niveles altos o superiores a lo normal ${ }^{11,25}$, trastorno de ansiedad generalizada ${ }^{27}$, trastorno mixto de ansiedad y depresión ${ }^{28}$, presentar síntomas del trastorno de estrés postraumático, 9,11,22,29-32, presentar trastornos de ansiedad en general ${ }^{28,33}$, ansiedad fóbical o en general manifestar síntomas de ansiedad. ${ }^{34-46}$ Depresión que incluye niveles altos de depresión ${ }^{11}$, presentación de trastorno depresivo ${ }^{26,28}$ o en general presentar síntomas. ${ }^{47}$ Estrés que incluye sentirse estresado ${ }^{19}$, presentación de síntomas de malestar peritraumático leve, moderado o severo ${ }^{9}$, fatiga ${ }^{16}$, estrés laboral ${ }^{44}$, altos niveles de activación ${ }^{44}$ o presentar síntomas de estrés en general. ${ }^{48-49}$ Sintomas psicóticos que incluyen paranoia ${ }^{13}$ y propensión al psicoticismo. ${ }^{1,13}$

Alteraciones del comportamiento como invertir un tiempo significativo en búsqueda de información sobre el COVID-19 $9^{18}$ y más probabilidades de establecer conductas obsesivo-compulsivas. ${ }^{1,13}$ Consumo de sustancias psicoactivas que incluye consumo de alcohol ${ }^{34,42}$, tabaco ${ }^{42}$ y remedios para dormir (medicamentos, suplementos, infusiones), entre otros. ${ }^{9}$

Sintomas fisiológicos (somáticos o psicosomáticos) que incluyen cefalea, faringitis, ${ }^{35}$ pérdida del apetito $\mathrm{y} \operatorname{tos}^{15}$ entre otros. ${ }^{13,38}$ Alteraciones del sueño con insomnio $^{18,29,30,35,36}$, letargo ${ }^{35}$, pobre calidad del sueño ${ }^{27}$, así como otros problemas de sueño. ${ }^{20}$

Algunos artículos evalúan el impacto psicológico como una medida general de las secuelas de la pandemia por COVID-19 en las personas o la población. ${ }^{45,48}$ Finalmente se resaltan cambios positivos en el bienestar descritos por algunos estudios, que incluyen aumento del apoyo o conexión social ${ }^{4,22}$, dedicar mayor tiempo al descanso y ejercicio, incremento del apoyo social y familiar ${ }^{17}$, trabajadores de la salud que se han inscrito voluntariamente en la primera línea ${ }^{44}$, oportunidad de reflexión, valorización del medio ambiente ${ }^{12}$, resiliencia ${ }^{22}$, mayor atención a la propia salud mental ${ }^{17} \mathrm{y}$ valorización de la Interdependencia y de los afectos. ${ }^{12}$ La anterior sintomatologia descrita se organiza de modo diverso en los diferentes grupos poblacionales, que fueron objeto de investigación en los estudios mecionados (tabla 2).

Tabla 2. Resultados por población y fases del brote

\begin{tabular}{|c|c|c|c|}
\hline Muestra & Fase inicial & Pico del brote & Después del pico \\
\hline Población general & $\begin{array}{l}\text { Ansiedad. } \\
\text { Consumo de sustancias (alcohol o tabaco). } \\
\text { Depresión } \\
\text { Estrés y fatiga. } \\
\text { Trastornos del sueño. } \\
\text { Preocupación por familiares. } \\
\text { Afectos negativos (incertidumbre, miedo, } \\
\text { angustia, soledad vinculada al encierro, } \\
\text { sensación de indefensión). } \\
\text { Valorización de la Interdependencia. } \\
\text { Oportunidad de reflexión. } \\
\text { Valorización del medio ambiente. } \\
\text { Valorización de los afectos. } \\
\text { Mayor atención y valor a la salud mental } \\
\text { personal. } \\
\text { Incremento del apoyo social y familiar. } \\
\text { Mayor tiempo al descanso y ejercicio. }\end{array}$ & $\begin{array}{l}\text { Depresión. } \\
\text { Afectos negativos (miedo, alto nivel de angustia } \\
\text { psicológica, sentirse abrumado y tenso). } \\
\text { Afectos positivos (calma). } \\
\text { Síntomas somáticos o psicosomáticos (tos y pérdida } \\
\text { de apetito). } \\
\text { Ansiedad y depresión). } \\
\text { Preocupación por familiares. } \\
\text { Preocupación financiera. } \\
\text { Miedo al COVID-19. } \\
\text { Aumento de la búsqueda de apoyo o conexión social. } \\
\text { Consumo de alcohol }\end{array}$ & $\begin{array}{l}\text { Estrés (malestar peritraumático leve, } \\
\text { moderado y severo trastorno de estrés } \\
\text { postraumático. } \\
\text { Depresión. } \\
\text { Ansiedad. } \\
\text { Consumo de sustancias psicoactivas. } \\
\text { Uso de remedios para dormir } \\
\text { (medicamentos, suplementos, } \\
\text { infusiones). } \\
\text { Percepción de deterioro de la salud } \\
\text { mental desde el inicio de la pandemia. } \\
\text { Preocupación por morir en caso de } \\
\text { contagio por COVID-19. } \\
\text { Afectos positivos (bienestar). } \\
\text { Afectos negativos (angustia). }\end{array}$ \\
\hline Personal sanitario & $\begin{array}{l}\text { Síntomas psicosomáticos. } \\
\text { Trastornos del sueño. } \\
\text { Depresión. } \\
\text { Ansiedad. } \\
\text { Estrés. } \\
\text { Innumerables trabajadores de la salud se } \\
\text { han inscrito voluntariamente en la primera } \\
\text { línea (altruismo). }\end{array}$ & $\begin{array}{l}\text { Ansiedad generalizada, estrés postraumático. } \\
\text { Estrés. } \\
\text { Depresión. } \\
\text { Alteración del sueño (pobre calidad). }\end{array}$ & $\begin{array}{l}\text { Estrés postraumático o síntomas de } \\
\text { estrés en general). } \\
\text { Depresión. } \\
\text { Ansiedad. } \\
\text { Alteración del sueño (insomnio). } \\
\text { Emociones negativas. }\end{array}$ \\
\hline
\end{tabular}




\begin{tabular}{|c|c|c|c|}
\hline Muestra & Fase inicial & Pico del brote & Después del pico \\
\hline $\begin{array}{l}\text { Personal sanitario } \\
\text { en primera línea }\end{array}$ & $\begin{array}{l}\text { Ansiedad. } \\
\text { Depresión. } \\
\text { Estrés. } \\
\text { Trastornos del sueño (letargo, insomnio, } \\
\text { entre otros). } \\
\text { Afectos negativos (emociones negativas, } \\
\text { miedo). } \\
\text { Síntomas somáticos o psicosomáticos } \\
\text { (dolor de cabeza y garganta). }\end{array}$ & $\begin{array}{l}\text { Síntomas psicosomáticos. } \\
\text { Síntomas obsesivos compulsivos. } \\
\text { Depresión. } \\
\text { Ansiedad (propensión a la ansiedad y a la ansiedad } \\
\text { fóbica). } \\
\text { Afectos negativos (terror, sensibilidad interpersonal, } \\
\text { hostilidad, rechazo inicial a la situación, miedo, } \\
\text { conmoción, sentimientos de impotencia). } \\
\text { Síntomas psicóticos (paranoia, propensión al } \\
\text { psicoticismo). } \\
\text { Tendencia a las conductas obsesivo-compulsivas. } \\
\text { Invertir un tiempo significativo en búsqueda de } \\
\text { información sobre el COVID-19. } \\
\text { Alteración del sueño (insomnio). }\end{array}$ & $\begin{array}{l}\text { Ansiedad. } \\
\text { Síntomas depresivos. } \\
\text { Estrés. }\end{array}$ \\
\hline $\begin{array}{l}\text { Personal sanitario } \\
\text { contagiado con } \\
\text { COVID-19 }\end{array}$ & & & $\begin{array}{l}\text { Ansiedad (estar nervioso). } \\
\text { Estrés (estrés psicológico). } \\
\text { Preocupaciones acerca de la salud de la familia } \\
\text { Cambios emocionales. }\end{array}$ \\
\hline $\begin{array}{l}\text { Estudiantes } \\
\text { primaria y/0 } \\
\text { secundaria }\end{array}$ & $\begin{array}{l}\text { Depresión. } \\
\text { Síntomas somáticos y psicosomáticos. } \\
\text { Ansiedad. }\end{array}$ & $\begin{array}{l}\text { Depresión. } \\
\text { Síntomas somáticos y psicosomáticos. } \\
\text { Ansiedad. }\end{array}$ & $\begin{array}{l}\text { Depresión. } \\
\text { Ansiedad. }\end{array}$ \\
\hline $\begin{array}{l}\text { Adultos jóvenes y } \\
\text { estudiantes } \\
\text { universitarios }\end{array}$ & $\begin{array}{l}\text { Impacto psicológico. } \\
\text { Depresión. } \\
\text { Ansiedad. } \\
\text { Estrés. }\end{array}$ & $\begin{array}{l}\text { Depresión. } \\
\text { Preocupaciones sobre COVID-19 con familiares. } \\
\text { Preocupaciones respecto al COVID-19 relacionadas } \\
\text { con ellos mismos. } \\
\text { Percepción de riesgo de infección. } \\
\text { Ansiedad (niveles superiores a lo normal, síntomas del } \\
\text { trastorno de estrés postraumático y de ansiedad en } \\
\text { general). } \\
\text { Estrés (niveles superiores a lo normal). } \\
\text { Afectos negativos (aburrimiento por estar sólo durante } \\
\text { el confinamiento y miedo a infectarse). } \\
\text { Tolerancia al estrés. } \\
\text { Resiliencia. } \\
\text { Aumento del apoyo o conexión social. }\end{array}$ & \\
\hline $\begin{array}{l}\text { Pacientes COVID } \\
\text { hospitalizados }\end{array}$ & $\begin{array}{l}\text { Ansiedad. } \\
\text { Depresión. } \\
\text { Estrés. }\end{array}$ & & $\begin{array}{l}\text { Estrés. } \\
\text { Ansiedad (niveles altos y síntomas de } \\
\text { estrés postraumático). } \\
\text { Afectos negativos (angustia). }\end{array}$ \\
\hline Adulto mayor & & & $\begin{array}{l}\text { Afectos negativos (presencia de } \\
\text { sentimientos negativos). } \\
\text { Alteración del sueño (problemas de sueño). } \\
\text { Ansiedad. }\end{array}$ \\
\hline $\begin{array}{l}\text { Sobrevivientes } \\
\text { COVID. }\end{array}$ & & $\begin{array}{l}\text { Ansiedad. } \\
\text { Depresión. } \\
\text { Afectos negativos. } \\
\text { Estrés. }\end{array}$ & \\
\hline $\begin{array}{l}\text { Personas que } \\
\text { regresan al trabajo }\end{array}$ & & & $\begin{array}{l}\text { Ansiedad. } \\
\text { Depresión. } \\
\text { Estrés. }\end{array}$ \\
\hline
\end{tabular}

Fuente: el autor

\section{DISCUSIÓN}

Durante el período en el cual se realizó la revisión de la literatura científica se vivía la primera ola de contagios por COVID-19 en Colombia y el mundo. Este tiempo estuvo marcado por la publicación de estudios que fijaban una sensación de incertidumbre en la generación de nuevos conocimientos, tanto para los escenarios estrictamente médicos y biológicos, como para aquellos contextos relacionados con la salud mental. Esta incertidumbre atrajo al escenario sanitario las tensiones y las posibilidades de la salud mental como elemento sustantivo para la vida humana, la calidad de vida y para todos los sistemas de salud en el mundo. En específico, la producción científica devela que los profesionales de la salud en primera línea de atención a pacientes con COVID-19, han presentado problemas de salud mental que afectan al sistema hospitalario y sanitario de los países. 
Las primeras publicaciones científicas reflejaron al inicio de la pandemia cómo se dinamizaron por primera vez sus efectos biológicos, sociales y psicológicos sobre las poblaciones. Así mismo, las publicaciones exponen las fortalezas en la producción académica de cada uno de los principales centros de investigación en el mundo. Para el caso colombiano, la búsqueda dejó ver la ausencia de producción científica en relación con el impacto de la pandemia en la salud mental de nuestra población durante la fase inicial del brote COVID-19.

\section{CONCLUSIONES}

La revisión de la literatura científica evidenció la aparición de síntomas psicopatológicos durante la pandemia del COVID-19 en la población mundial, en especial agrupados en las categorías diagnósticas de ansiedad, depresión y estrés. Durante el desarrollo del estudio se diseñó una matriz de selección y evaluación de la información, que permitió la organización y síntesis del material en cinco pasos, incluyendo en uno de ellos la evaluación de la calidad metodológica y con ello permitiendo la identificación de diferentes sintomatologías en salud mental durante el brote del COVID-19.

El estudio reveló investigaciones principalmente cuantitativas donde se destaca la afectación a la población en general y en especial a los profesionales de la salud de primera línea de atención en COVID-19, como grupo poblacional de alto riesgo psicológico. Dentro de los 43 estudios seleccionados no se encontraron investigaciones colombianas. Al tiempo se destaca a China como el país con mayor producción científica en esta área para el período de búsqueda de la información.

\section{CONSIDERACIONES ÉTICAS}

Se trabajó sobre fuentes secundarias de información, garantizando el respeto a los derechos de autor siguiendo la Ley 23 de 1982 "Sobre Derechos de Autor"

\section{CONFLICTOS DE INTERÉS}

Los autores no declaran conflictos de interés.

\section{AGRADECIMIENTOS}

A los estudiantes del semillero GIPSAD, con la sublínea de desarrollo y sociedad, que con su compromiso, dedicación y empeño permitieron la culminación de este estudio y probablemente harán aportes muy importantes a las siguientes investigaciones de la línea de investigación en la temática.

\section{REFERENCIAS}

1. Xing J, Sun N, Xu J, Geng S, Li Y. Study of the mental health status of medical personnel dealing with new coronavirus pneumonia. PLoS One. 2020;15(5):e0233145. doi: 10.1371/journal. pone.0233145.

2. Wang C, Horby PW, Hayden FG, Gao GF. A novel coronavirus outbreak of global health concern. Lancet. 2020;395(10223):470473. doi: 10.1016/S0140-6736(20)30185-9. Epub 2020 Jan 24. Erratum in: Lancet. 2020.

3. Zhang Y, Zhang H, Ma X, Di Q. Mental Health Problems during the COVID-19 Pandemics and the Mitigation Effects of Exercise: A Longitudinal Study of College Students in China. Int J Environ Res Public Health. 2020;17(10):3722. doi: 10.3390/ ijerph17103722.

4. Tull MT, et al. Psychological Outcomes Associated With Stayat-Home Orders and the Perceived Impact of COVID-19 On Daily Life. Psychiatry Res. 2020;289:113098. doi: 10.1016/j. psychres.2020.113098

5. Elbay RY, Kurtulmu囚 A, Arpacio囚lu S, Karadere E. Depression, anxiety, stress levels of physicians and associated factors in Covid-19 pandemics. Psychiatry Res. 2020 May 27;290:113130. doi: 10.1016/j.psychres.2020.113130

6. Guirao Goris Silamani JA. Utilidad y tipos de revisión de literatura. Ene. 2015; 9(2). doi: 10.4321/S1988-348X2015000200002

7. von Elm E, Altman DG, Egger M, Pocock SJ, Gøtzsche PC, Vandenbroucke JP, et al. Declaración de la Iniciativa STROBE (Strengthening the Reporting of Observational studies in Epidemiology): directrices para la comunicación de estudios observacionales. Gac Sanit. 2008;22(2):144-50. https://doi. org/10.1157/13119325

8. Garcés Cano EJ, Duque Oliva, EJ. Metodología para el análisis y la revisión crítica de artículos de investigación. Revista Innovar. 2007;17(29):184-194.

9. Costantini A, Mazzotti E. Italian validation of CoViD-19 Peritraumatic Distress Index and preliminary data in a sample of general population. Riv Psichiatr. 2020;55(3):145-151. doi: $10.1708 / 3382.33570$

10. Gómez-Salgado, J, Andrés-Villas M, Domínguez-Salas S, DíazMilanés D, Ruiz-Frutos C. Related Health Factors of Psychological Distress During the COVID-19 Pandemic in Spain. Int J Environ Res Public Health. 2020;17(11):3947. doi: 10.3390/ijerph17113947

11. Guo Q, Zheng Y, Shi J, Wang J, Li G, Li C, Fromson JA, Xu Y, Liu X, Xu H, Zhang T, Lu Y, Chen X, Hu H, Tang Y, Yang S, Zhou H, Wang $X$, Chen H, Wang Z, Yang Z. Immediate psychological distress in quarantined patients with COVID-19 and its association with peripheral inflammation: A mixed-method study. Brain Behav Immun. 2020;88:17-27. doi:10.1016/j.bbi.2020.05.038

12. Johnson MC, Saletti-Cuesta L, Tumas N. Emociones, preocupaciones y reflexiones frente a la pandemia del COVID-19 en Argentina. Ciênc. saúde coletiva. 2020;25(Suppl1):2447-2456. doi: 10.1590/1413-81232020256.1.10472020 
13. Wu K, Wei X. Analysis of Psychological and Sleep Status and Exercise Rehabilitation of Front-Line Clinical Staff in the Fight Against COVID-19 in China. Med Sci Monit Basic Res. 2020;26:e924085. doi: 10.12659/MSMBR.924085.

14. Hong,Yan., Cao, H.,Leung, D., y Mak, Y. The Psychological Impacts of a COVID-19 Outbreak on College Students in China: A Longitudinal Study. Int J Environ Res Public Health. 2020;17(11):3933. doi: 10.3390/ijerph17113933

15. Olufadewa I., Adesina M, Oladokun B, Baru A, Oladele R, Iyanda T, Ajibade O, Abudu, F. "I Was Scared I Might Die Alone": A Qualitative Study on the Physiological and Psychological Experience of COVID-19 Survivors and the Quality of Care Received at Health Facilities. Int J Travel Med Glob Health. 2020;8(2):51-57. doi: 10.34172/ijtmgh.2020.09

16. Sun N, Wei L, Shi S, Jiao D, Song R, Ma L, Wang H, Wang C, Wang Z, You Y, Liu S, Wang H. A qualitative study on the psychological experience of caregivers of COVID-19 patients. Am J Infect Control. 2020;48(6):592-598. doi: 10.1016/j.ajic.2020.03.018.

17. Yingfei Zhang, Zheng Feei Ma. Impact of the COVID-19 Pandemic on Mental Health and Quality of Life among Local Residents in Liaoning Province, China: A Cross-Sectional Study. Int J Environ Res Public Health. 2020;17(7):2381. doi: 10.3390/ ijerph17072381.

18. Amerio A, Bianchi D, Santi F, Costantini L, Odone A, Signorelli C, Costanza A, Serafini G, Amore M, Aguglia A. Covid-19 pandemic impact on mental health: a web-based cross-sectional survey on a sample of Italian general practitioners. Acta Biomed. 2020;91(2):83-88. doi: 10.23750/abm.v9li2.9619

19. Madani A, Boutebal SE, Bryant CR. The Psychological Impact of Confinement Linked to the Coronavirus Epidemic COVID-19 in Algeria. Int J Environ Res Public Health. 2020;17(10):3604. doi: 10.3390/ijerph17103604.

20. Goodman-Casanova JM, Dura-Perez E, Guzman-Parra J, CuestaVargas A, Mayoral-Cleries F. Telehealth Home Support During COVID-19 Confinement for Community-Dwelling Older Adults With Mild Cognitive Impairment or Mild Dementia: Survey Study. J Med Internet Res. 2020;22(5):e19434. doi: 10.2196/19434.

21. Smith L, Jacob L, Yakkundi A, McDermott D, Armstrong NC, Barnett Y, López-Sánchez GF, Martin S, Butler L, Tully MA. Correlates of symptoms of anxiety and depression and mental wellbeing associated with COVID-19: a cross-sectional study of UK-based respondents. Psychiatry Res. 2020;291:113138. doi: 10.1016/j.psychres.2020.113138.

22. Liu CH, Zhang E, Wong GTF, Hyun S, Hahm HC. Factors associated with depression, anxiety, and PTSD symptomatology during the COVID-19 pandemic: Clinical implications for U.S. young adult mental health. Psychiatry Res. 2020;290:113172. doi: 10.1016/j. psychres.2020.113172.

23. Bitan DT, Giron A, Bloch Y, Mayer Y, shiffman N, Mendlovic S. Fear of COVID-19 scale: Psychometric characteristics, reliability and validity in the Israeli population. Psychiatry Res. 2020;289:113100. doi: 10.1016/j.psychres.2020.113100.
24. Jin YH, Huang Q, Wang YY, Zeng XT, Luo LS, Pan ZY, Yuan YF, Chen ZM, Cheng ZS, Huang X, Wang N, Li BH, Zi H, Zhao MJ, Ma LL, Deng T, Wang Y, Wang XH. Perceived infection transmission routes, infection control practices, psychosocial changes, and management of COVID-19 infected healthcare workers in a tertiary acute care hospital in Wuhan: a cross-sectional survey. Version 2. Mil Med Res. 2020;7(1):24. doi: 10.1186/s40779-020-00254-8.

25. Germani A, Buratta L, Delvecchio E, Mazzeschi C. Emerging Adults and COVID-19: The Role of Individualism-Collectivism on Perceived Risks and Psychological Maladjustment. Int J Environ Res Public Health. 2020;17(10):3497. doi: 10.3390/ijerph17103497.

26. Choi, E.P.H.; Hui, B.P.H.; Wan, E.Y.F. Depression and Anxiety in Hong Kong during COVID-19. Int. J. Environ. Res. Public Health 2020;17(10):3740. doi: 10.3390/ijerph17103740

27. Huang Y, Zhao N. Generalized anxiety disorder, depressive symptoms and sleep quality during COVID-19 outbreak in China: a web-based cross-sectional survey. Psychiatry Res. 2020;288:112954. doi: 10.1016/j.psychres.2020.112954.

28. Gao J, Zheng P, Jia Y, Chen H, Mao Y, Chen S, Wang Y, Fu H, Dai J. Mental health problems and social media exposure during COVID-19 outbreak. PLoS One. 2020;15(4):e0231924. doi: 10.1371/ journal.pone.0231924.

29. Guo J, Feng XL, Wang XH, van IJzendoorn MH. Coping with COVID-19: Exposure to COVID-19 and Negative Impact on Livelihood Predict Elevated Mental Health Problems in Chinese Adults. Int J Environ Res Public Health. 2020;17(11):3857. doi: 10.3390/ijerph17113857.

30. Rossi R, Socci V, Pacitti F, Di Lorenzo G, Di Marco A, Siracusano A, Rossi A. Mental Health Outcomes Among Frontline and Second-Line Health Care Workers During the Coronavirus Disease 2019 (COVID-19) Pandemic in Italy. JAMA Netw Open. 2020;3(5):e2010185. doi: 10.1001/ jamanetworkopen.2020.10185.

31. Song, X., Fu, W., Liu, X., Luo, Z., Wang, R., Zhou, N., Yan, S., \& Lv, C. Mental health status of medical staff in emergency departments during the Coronavirus disease 2019 epidemic in China. Brain Behav Immun. 2020;88:60-65. doi: 10.1016/j.bbi.2020.06.002

32. Wanqiu, T., Fengyi, H., Roger S. M., Li, J., Xiaojiang, J., Ling Zhang, Xinling, Z., Yiran, Z., Yirong, H., Xi, L., Zhisong, Z., Andre, L., Roger,H., Bach, T., Cyrus, H., y Wilson, T. Is returning to work during the COVID-19 pandemic stressful? A study on immediate mental health status and psychoneuroimmunity prevention measures of Chinese workforce. Brain, Behavior, and Immunity. 2020.87:84-92. https://doi.org/10.1016/j.bbi.2020.04.055

33. Liu CY, Yang YZ, Zhang XM, Xu X, Dou QL, Zhang WW, Cheng ASK. The prevalence and influencing factors in anxiety in medical workers fighting COVID-19 in China: a cross-sectional survey. Epidemiol Infect. 2020;148:e98. doi: 10.1017/S0950268820001107.

34. Ahmed MZ, Ahmed O, Aibao Z, Hanbin S, Siyu L, Ahmad A. Epidemic of COVID-19 in China and associated Psychological Problems. Asian J Psychiatr. 2020;51:102092. doi: 10.1016/j. ajp.2020.102092. 
35. Chew NWS, Lee GKH, Tan BYQ, Jing M, Goh Y, Ngiam NJH, Yeo LLL, Ahmad A, Ahmed Khan F, Napolean Shanmugam G, Sharma AK, Komalkumar RN, Meenakshi PV, Shah K, Patel B, et al. A multinational, multicentre study on the psychological outcomes and associated physical symptoms amongst healthcare workers during COVID-19 outbreak. Brain Behav Immun. 2020;88:559565. doi: 10.1016/j.bbi.2020.04.049.

36. Lai J, Ma S, Wang Y, Cai Z, Hu J, Wei N, Wu J, Du H, Chen T, Li R, Tan H, Kang L, Yao L, Huang M, Wang H, Wang G, Liu Z, Hu S. Factors Associated With Mental Health Outcomes Among Health Care Workers Exposed to Coronavirus Disease 2019. JAMA Netw Open. 2020;3(3):e203976. doi: 10.1001/jamanetworkopen.2020.3976.

37. Lei L, Huang X, Zhang S, Yang J, Yang L, Xu M. Comparison of Prevalence and Associated Factors of Anxiety and Depression Among People Affected by versus People Unaffected by Quarantine During the COVID-19 Epidemic in Southwestern China. Med Sci Monit. 2020;26:e924609. doi: 10.12659/MSM.924609.

38. Liu S, Liu Y, Liu Y. Somatic symptoms and concern regarding COVID-19 among Chinese college and primary school students: A cross-sectional survey. Psychiatry Res. 2020;289:113070. doi: 10.1016/j.psychres.2020.113070.

39. Liu X, Luo WT, Li Y, Li CN, Hong ZS, Chen HL, Xiao F, Xia JY. Psychological status and behavior changes of the public during the COVID-19 epidemic in China. Version 2. Infect Dis Poverty. 2020 May;9(1):58. doi: 10.1186/s40249-020-00678-3.

40. Odriozola-González P, Planchuelo-Gómez Á, Irurtia MJ, de Luis-García R. Psychological effects of the COVID-19 outbreak and lockdown among students and workers of a Spanish university. Psychiatry Res. 2020;290:113108. doi: 10.1016/j. psychres.2020.113108.

41. Ozamiz-Etxebarria, Naiara et al. Niveles de estrés, ansiedad y depresión en la primera fase del brote del COVID-19 en una muestra recogida en el norte de España. Cadernos de Saúde Pública [online], 2020, 36(4) [Accessed 30 June 2020] , e00054020. Available from: <https://doi.org/10.1590/0102-311X00054020>. ISSN 1678-4464. https://doi.org/10.1590/0102-311X00054020.
42. Stanton R, To QG, Khalesi S, et al. Depression, anxiety and stress during COVID-19: Associations with changes in physical activity, sleep, tobacco and alcohol use in australian adults. Int J Environ Res Public Health. 2020;17(11):4065. doi: 10.3390/ijerph17114065

43. Steven Taylor, Caeleigh A. Landry, Michelle M. Paluszek, Thomas A. Fergus, Dean McKay, Gordon J.G. Asmundson. Development and initial validation of the COVID Stress Scales. J Anxiety Disord. 2020; 72:102232. https://doi.org/10.1016/j.janxdis.2020.102232.

44. Sun D, Yang D, Li Y, Zhou J, Wang W, Wang Q, Lin N, Cao A, Wang H, Zhang Q. Psychological impact of 2019 novel coronavirus (2019-nCoV) outbreak in health workers in China. Epidemiol Infect. 2020;148:e96. doi: 10.1017/S0950268820001090.

45. Wang C, Pan R, Wan X, Tan Y, Xu L, McIntyre RS, Choo FN, Tran B, Ho R, Sharma VK, Ho C. A longitudinal study on the mental health of general population during the COVID-19 epidemic in China. Brain Behav Immun. 2020;87:40-48. doi: 10.1016/j. bbi.2020.04.028.

46. Yang S, Kwak SG, Ko EJ, Chang MC. The Mental Health Burden of the COVID-19 Pandemic on Physical Therapists. Int J Environ Res Public Health. 2020;17(10):3723. doi: 10.3390/ijerph17103723.

47. Wang H, Xia Q, Xiong Z, Li Z, Xiang W, Yuan Y, Liu Y, Li Z. The psychological distress and coping styles in the early stages of the 2019 coronavirus disease (COVID-19) epidemic in the general mainland Chinese population: A web-based survey. PLoS One. 2020;15(5):e0233410. doi: 10.1371/journal.pone.0233410.

48. Varshney M, Parel JT, Raizada N, Sarin SK. Initial psychological impact of COVID-19 and its correlates in Indian Community: An online (FEEL-COVID) survey. PLoS One. 2020;15(5):e0233874. doi: 10.1371 /journal.pone.0233874.

49. Ley 23 de 1982. Sobre derechos de autor. Colombia: Congreso de la República; 1982. 